\title{
Endoscopic Band Ligation Compared With Endoscopic Submucosal Dissection For Removal of Muscularis Propria Originated Small Neoplasms
}

\author{
Qunqun $\mathrm{Ye}^{1,2}$ and Chunxiao Chen*1 \\ ${ }^{1}$ Department of Gastroenterology, China \\ ${ }^{2}$ Department of Gastroenterology, Taiwan \\ *Corresponding author: Chunxiao Chen, Department of Gastroenterology, China
}

\section{ARTICLE INFO}

Received: 蔧 March 01, 2019

Published: 幽 March 11, 2019

Citation: Qunqun Ye, Chunxiao Chen. Endoscopic Band Ligation Compared With Endoscopic Submucosal Dissection For Removal of Muscularis Propria Originated Small Neoplasms. Biomed J Sci \& Tech Res 15(4)-2019. BJSTR. MS.ID.002746.

Abbreviations: EUS: Endoscopic Ultrasonography; GIST: Gastrointestinal Stromal Tumors; EBL : Endoscopic Band Ligation; ESD: Endoscopic Submucosal Dissection; EFR: Endoscopic Full-Thickness Resection; SMTs: Submucosal Tumors; GISTs: Gastrointestinal Stromal Tumors
ABSTRACT

Aim: To compare efficacy and safety between endoscopic band ligation (EBL) and endoscopic submucosal dissection (ESD) in treatment of muscularis propria originated small neoplasms $(<1.2 \mathrm{~cm})$.

Methods: A total of 105 patients receiving endoscopic ultrasonography (EUS) and diagnosed muscularis propria originated small neoplasms $(<1.2 \mathrm{~cm})$ were included in this retrospective study. Patients were divided into two groups, EBL $(n=51)$ and ESD $(n=54)$. Clinical data, peri-procedure outcome and follow-up conditions were collected and analyzed.

Results: Forty-nine patients $(96.1 \%)$ in EBL successfully completed endoscopic procedures. Of the 49 patients adopted EBL, 1 patient developed post-operation perforation and 2 patients complicated with minor bleeding. During follow-up period ( 2 weeks- 12 months), all 49 neoplasms fell off, with none relapsed. In ESD group, 52 patients (96.3\%) successfully completed endoscopic procedure and 2 patients switched to open surgery for adhesion. Fourteen patients went through initiative perforation. None relapsed during follow-up (3 months-12 months). As for post-procedure complications, the two groups showed similar rate $(6.1 \%, 3 / 49$ vs. $0 \%, 0 / 52, \mathrm{P}=0.070)$. EBL group showed significantly shorter operating time $(16.2 \pm 3.7 \mathrm{~min}$ vs. $73.2 \pm 36.4 \mathrm{~min}, \mathrm{P}=0.001)$, post-procedure fasting time $(1.3 \pm 0.7 \mathrm{~d}$ vs. $3.8 \pm 1.4 \mathrm{~d}, \mathrm{P}=0.001)$, hospital stay $(2.8 \pm 0.9 \mathrm{~d}$ vs. $5.7 \pm 1.4 \mathrm{~d}, \mathrm{P}=0.001)$, and lower cost ( $9000 ¥ \pm 3000 ¥$ vs. $19000 ¥ \pm 3000 ¥, \mathrm{P}=0.001)$.

Conclusion: For small neoplasms originated from muscularis propria, EBL showed better safety, efficacy and cost-effectiveness compared with ESD.

Keywords: Muscularis Propria Neoplasm; Endoscopic Band Ligation; Endoscopic Submucosal Dissection

\section{Introduction}

With rapid development of endoscopic ultrasonography (EUS), detecting rate of upper digestive tract muscularis propria originated small neoplasms is increasing. Histologically, muscularis propria originated neoplasms are divided into gastrointestinal stromal tumors (GIST), liomyoma, schwannoma and calcified fibrous tumor. Though for most small neoplasms, regular endoscopic followup shows lower risk, but patients would develop great mental burden. Taking into carcinogenesis potential of GIST and liomyoma, endoscopic procedures with safety, efficacy and convenience were now back to field. As for endoscopic procedures of such lesions, endoscopic band ligation (EBL) and endoscopic submucosal dissection (ESD) have been widely used now. ESD is now widely used in submucosal lesion dissection, but with limitation of relative long learning period, complicated procedures. Compared with 
ESD, EBL shows better convenience, but lacks pathological studies for random discharge of neoplasms. So herein, we conducted a retrospective case-control study to investigate the treatment value of ESD and EBL in muscularis propria originated small neoplasms.

\section{Materials and Methods}

\section{Subjects}

A total of 105 patients diagnosed as neoplasms originated from muscularis propria by endoscopic with ultrasonography and then underwent ESD and EBL from Oct, 2013 to Jun, 2015 were included in the study. Informed consent was obtained from all patients for being included in the study. Exclusion criteria were:

a) Muscularis propria derived neoplasm $>1.2 \mathrm{~cm}$;

b) Complicated with other endoscopic lesion;

c) Anti-neoplasm therapy during endoscopic procedure;

d) Age $>80$ years or $<18$ years;

e) Low compliance;

f) Other situation that may influence result.

\section{Pre-Procedure Evaluation}

Patients underwent gastric endoscopy, endoscopic ultrasonography, abdominal CT scan for pre-procedure evaluation of size, location and type of neoplasm. And routine chest X-ray, electrocardiogram, pulmonary function, blood routine, biochemical analysis, coagulation function were completed before procedure.

\section{Endoscopic Procedures}

For EBL group, routine procedures were as follows: place ligation device on top of endoscope, insert endoscope close to lesion, choose a suitable place and angle, put lesion into transparent cap and suck with vacuum till whole lesion into transparent cap, and release one or two rubber band after 30 seconds of forced suction. Repeat the above procedure if necessary to ensure ligation of the lesion. Titanium clip might be use if necessary. For ESD group, routine procedures were as follows: Mark outer $0.5 \mathrm{~cm}$ margin of lesion with argon beam coagulator, inject into submucosa with dying solution (2-3ml methylene blue, $1 \mathrm{ml}$ epinephrine and $100 \mathrm{ml}$ saline), dissect with Hook knife to expose lesion, strip lesion with IT knife or Hook knife even with perforation, argon beam coagulator to deal with bleeding in the surface, and close wound with titanium clip or with purse string suture.

\section{Post-Procedure Treatment and Follow-Up}

For EBL, fast for one day after procedure, and routine antiacid therapy, mucous protection agent for two weeks. Repeat endoscopic ultrasonography or endoscopy for 2 weeks or 3 months. For ESD group, fast, gastrointestinal decompression, anti-acid and parenteral nutrition for two to three days as for routine patients. The time might be extended to one week for endoscopic full-thickness resection (EFR) patients, and anti-biotics might be used in addition. Routine anti-acid therapy, mucous protection agent were used till two months and followed up with endoscopic ultrasonography or endoscopy for 3 months to 1 year.

\section{Statistical Analysis}

Data collected included demographic, clinical data as well as operating time, post-operate fast time, hospital stay and cost. Normality of distribution was tested with Kolmogorov-Smirnov test. Normally distributed variables were presented as mean \pm standard deviation (SD); variables with skewed distributions were presented as median (interquartile range). Student's t-test or MannWhitney $U$ test for continuous variables, and $\chi 2$ test for categorical variables were used to compare the parameters between cases and controls. Statistical analyses were performed using SPSS 20.0 (SPSS Inc., Chicago, IL, USA). A two-sided P $<0.05$ was considered statistically significant.

\section{Results}

\section{Clinical Evaluations}

A total of 105 patients were included in our study, 54 in ESD group and 51 in EBL group. Two groups showed no difference in age, sex, tumor size ( $\mathrm{P}>0.05$ for all) (Table 1). 59 patients (56.2\%) complaint with abdominal discomfort, distension, abdominal pain. 46 patients $(43.8 \%)$ were random findings during health examination. As for tumor location, 52 (49.5\%) were in the fundus, $51(48.6 \%)$ in gastric body, $2(1.9 \%)$ in antrum. Pathological analysis was successfully done for all patients in ESD group. 24 were diagnosed with interatitialoma, with 21 being extreme low risk, 3 as low risk, 23 liomyoma, 3 Schwannoma, 2 ectopic pancreas, 1 fibroadenoma, 1 neuroendocrine neoplasm (Table 1).

Table 1: Demographic data in endoscopic submucosal dissection and endoscopic band ligation group.

\begin{tabular}{|c|c|c|c|}
\hline Variable & Endoscopic Band Ligation & Endoscopic Submucosal Dissection & $P$ value \\
\hline Gender (Male, $\%)$ & $21(41 \%)$ & $17(31 \%)$ & 0.301 \\
\hline Age (year) & $54.04 \pm 10.31$ & $51.57 \pm 12.93$ & 0.284 \\
\hline Tumor size (cm) & $0.841 \pm 0.20$ & $0.857 \pm 0.29$ & 0.747 \\
\hline Tumor location & & & 0.259 \\
\hline Fundus & 25 & 30 & \\
\hline Body & 26 & 22 & \\
\hline Antrum & 0 & 0 & \\
\hline
\end{tabular}




\section{Endoscopic Procedures}

49 patients (96.1\%) in EBL group successfully completed endoscopic procedures. Two aborted for not obvious occupation during endoscopy and discharged for follow-up. 52 patients (96.3\%) completed ESD. Two switched to surgery for severe adhesion. 14 underwent EFR for to fully resect tumor. 12 used titanium clip to close perforation and two used purse string suture as well.

\section{Peri-Procedure Comparison}

One patient in EBL group complicated with acute perforation, and successfully treated with purse string suture; two had minor bleeding after procedure and treated conservatively. 13 patients in ESD group had fever after procedure, but none complicated with gastric intestinal bleeding or severe infection or even death. Post-procedure complication rate showed no significant difference between two groups ( $\mathrm{P}>0.05$ for all). But as for procedure time, post-procedure fast time, hospital stay and cost, EBL group were all lower ( $\mathrm{P}<0.05$ for all,) (Table 2).

\section{Follow-Up Conditions}

Follow up duration in EBL group varied from 2 weeks to one year, and none of 49 patients showed recurrence. As for ESD group, post-procedure endoscopy in three to 12 months after procedure was all negative as well.

Table 2: Peri-procedure comparison between endoscopic submucosal dissection and endoscopic band ligation group.

\begin{tabular}{|c|c|c|}
\hline Variable & Endoscopic Band Ligation Group & Endoscopic Submucosal Dissection Group \\
\hline Number, n & 49 & 52 \\
\hline Procedure duration (min) & $16.2 \pm 3.7$ & $73.2 \pm 36.4$ \\
\hline Post-procedure fast time (days) & $1.3 \pm 0.7$ & $3.8 \pm 1.4$ \\
\hline Hospital stay (days) & $2.8 \pm 0.9$ & $5.7 \pm 1.4$ \\
\hline Total cost (thousand yuan) & $9 \pm 3$ & 0.001 \\
\hline Post-procedure complications (n) & 3 & 0.001 \\
\hline Antrum & 0 & 0.0701 \\
\hline
\end{tabular}

\section{Discussion}

This study compared EBL and ESD procedure in treatment of small neoplasms originated from muscularis propria. Both groups showed similar peri-procedure complications. EBL group had lower procedure time, post-procedure fast time, and hospital stay and cost as well. Submucosal tumors (SMTs) are common neoplasms derived from muscularis propria, which are mainly leiomyomas and gastrointestinal stromaltumors (GISTs). Leiomyomas are usually benign. GISTs can be recurrent and metastasis. Whether it's benign or malignant is decided by size and mitosis index [1] for example, tumor less than $2 \mathrm{~cm}$ with MI less than 5/50 high power field was known as benign. GISTs are relatively rare endoscopic findings, with prevalence of 1-2/100,000. Surgery or endoscopic procedure is common treatment for GISTs. The way of GISTs treatment is decided by location and invasion deepness of the tumor, rather than histological type. Most GISTs are non-invasive and seldom showed lymph node metastasis. So, lymphadenectomy is not usually necessary. For now, treatment of SMTs less than $2 \mathrm{~cm}$ is not consistent. Some experts recommend close endoscope follow up for its low invasiveness. But as endoscopy technology developed, endoscopy treatment of GISTs is of great value, such as ESD, EMR, EBl et al. [2,3].

EBL is newly developed procedure for GISTs. Similar as that in treatment of esophageal varix, EBL included processes of suction, ligation, release of band and finally led to tumor ischemia, falling off and heal. Sun, et al reported that their experience in EBL for small leiomyoma [4]. EBL showed better accessibility, convenience, less trauma, shorter hospital stay and lower cost [4]. EBL is relatively safe that after ligation of whole layer of stomach, tumor would fall off after ischemia and necrosis, and left ulcer will be healed in a short period. However, in our study, one patient in EBL group had acute perforation after procedure, might be owing to early fall off of band. In order to avoid such complication, titanium clip can be used. As diameter of transparent cap is limited, for tumor greater than $12 \mathrm{~mm}$, complete suction was hard, so longer suction time is needed [5]. One limitation for EBL is that usually tumor fall of time is long and random, so pathological analysis is not applicable. In our study, ESD group showed $44.4 \%$ GISTs, $42.6 \%$ liomyoma, which all turned to be low or extreme low degree.

So, clinically, close follow up is needed to ensure fall off of tumor as well as recurrence and in our study, after 2 weeks to 12 months post-procedure follow up, all tumors were off, and none had recurrence. ESD is now widely used endoscopic technology for dissection of muscularis propria tumors with application of endoscopy, knife, injecting needle, transparent cap, titanium clip, coagulation clamp. Advantage of ESD is ability to gain pathological evidence. As endoscopic skills and equipment develop rapidly, for tumors invaded into serosa, EFR is also safe and effective [6]. Once perforation happened, titanium clip can be used to close small wound $(<3 \mathrm{~cm})$, while larger wound would be closed by purse string suture [7]. In our study, 14 patients ender went EFR, adopted purse string suture and none switched to surgery or had post-procedure bleeding. For muscularis propria tumor less than $3 \mathrm{~cm}$, compared with surgery, ESD has less trauma, lower hospital stay and quick recovery [8]. 


\section{Conclusion}

In conclusion, as for small neoplasm derived from muscularis propria, compared with EBL, ESD group showed lower procedure time, post-procedure fast time, hospital stay with no increase in peri-procedure complications.

\section{References}

1. Bertolini V, Chiaravalli AM, Klersy C, Placidi C, Marchet S, et al. (2008) Gastrointestinal stromal tumors--frequency, malignancy, and new prognostic factors: the experience of a single institution. Pathol Res Pract 204(4): 219-233.

2. Ono H, Kondo H, Gotoda T, Shirao K, Yamaguchi H, et al. (2001) Endoscopic mucosal resection for treatment of early gastric cancer. Gut 48(2): 225-229.

3. Ponchon T (2001) Endoscopic mucosal resection. J Clin Gastroenterol 32: 6-10.

\section{ISSN: 2574-1241}

DOI: 10.26717/BJSTR.2019.15.002746

Chunxiao Chen. Biomed J Sci \& Tech Res

(C) This work is licensed under Creative

Submission Link: https://biomedres.us/submit-manuscript.php
4. Sun S, Jin Y, Chang G, Wang C, Li X, et al. (2004) Endoscopic band ligation without electrosurgery: a new technique for excision of small upper-GI leiomyoma. Gastrointest Endosc 60(2): 218-222.

5. Xiang L, Siyu S, Nan G, Sheng W, Qingjie LU, et al. (2008) The therapeutic value of endoscopic band ligation in gastric stromal tumors. Chinese Journal of Digestive Endoscopy 25: 236-238.

6. Jianhua J, Xueliang L, Lianzhen Y, Shuping Y, Ruihua S (2011) Endoscopic full-thickness resection for gastric stromal tumor. Chinese Journal of Digestive Endoscopy 28: 632-634.

7. Sumiyama K, Gostout CJ, Rajan E, Bakken TA, Deters JL (2007) Endoscopic full-thickness closure of large gastric perforations by use of tissue anchors. Gastrointest Endosc 65(1): 134-139.

8. Fuchao L, Dongtao S, Xiaojun Z, Rui L, Zhongqi M, et al. (2014) Comparison between endoscopy and laparoscopy in resection of gastric stromal tumor. Chinese Journal of Digestive Endoscopy 31: 69-71.

$\begin{array}{ll}\text { BIOMEDICAL } & \text { Assets of Publishing with us } \\ \text { RESEARCHES } & \text { - Global archiving of articles } \\ & \text { - Immediate, unrestricted online access } \\ & \text { - Rigorous Peer Review Process } \\ \end{array}$

